2 West Nile Virus Subverts T Cell Stimulatory Capacity of

\title{
3 Human Dendritic Cells
}

4 Matthew G. Zimmerman ${ }^{1,2, \S}$, James R. Bowen ${ }^{1,2, \S}$, Circe E. McDonald ${ }^{1,2}$, Bali

5 Pulendran ${ }^{2,3}$, Mehul S. Suthar ${ }^{1,2, *}$

$7 \quad{ }^{1}$ Department of Pediatrics, Division of Infectious Diseases, Emory University School of

8 Medicine, Atlanta, GA 30322, USA.

92 Emory Vaccine Center, Yerkes National Primate Research Center, Atlanta, GA 30329,

10 USA.

$11{ }^{3}$ Department of Pathology and Laboratory Medicine, Emory University School of

12 Medicine, Atlanta, GA 30329, USA

13 §These authors contributed equally to this work

14 *Correspondence: msuthar@emory.edu (M.S.S.) 


\section{Abstract}

16 West Nile virus (WNV) is a neurotropic flavivirus and the leading cause of mosquito-

17 borne encephalitis in the United States. Recent studies in humans have found that

18 dysfunctional T cell responses strongly correlate with development of severe WNV

19 neuroinvasive disease. However, the contributions of human dendritic cells (DCs) in

20 priming WNV-specific $\mathrm{T}$ cell immunity remains poorly understood. Here, we

21 demonstrate that human monocyte-derived DCs (moDCs) support productive viral

22 replication following infection with a pathogenic strain of WNV. Antiviral effector gene

23 transcription was strongly induced during the log-phase viral growth, while secretion of

24 type I interferons (IFN) occurred with delayed kinetics. Activation of RIG-I like receptor

25 (RLR) or type I IFN signaling prior to log-phase viral growth significantly diminished viral

26 replication, suggesting that activation of antiviral programs early can block WNV

27 infection. In contrast to the induction of antiviral responses, WNV infection did not

28 promote transcription or secretion of pro-inflammatory (IL-6, GM-CSF, CCL3, CCL5,

29 CXCL9) or T cell modulatory cytokines (IL-4, IL-12, IL-15). There was also minimal

30 induction of molecules associated with antigen presentation and $\mathrm{T}$ cell priming,

31 including the co-stimulatory molecules CD80, CD86, and CD40. Functionally, WNV-

32 infected moDCs dampened allogenic CD4 and CD8 T cell activation and proliferation.

33 Combined, we propose a model where WNV subverts human DC activation to

34 compromise priming of WNV-specific T cell immunity. 


\section{Importance}

$40 \quad$ West Nile virus (WNV) is an encephalitic flavivirus that remains endemic in the

41 United States. Previous studies have found dysfunctional T cell responses correlate to

42 severe disease outcomes during human WNV infection. Here, we sought to better

43 understand the ability of WNV to program human dendritic cells (DCs) to prime WNV-

44 specific T cell responses. While productive infection of monocyte-derived DCs activated

45 antiviral and type I interferon responses, molecules associated with inflammation and

46 programming of $\mathrm{T}$ cells were minimally induced. Functionally, WNV-infected DCs

47 dampened T cell activation and proliferation during an allogeneic response. Combined,

48 our data supports a model where WNV infection of human DCs compromises WNV-

49 specific T cell immunity. 


\section{Introduction}

West Nile virus (WNV) is a neurotropic flavivirus that remains the leading cause

52 of mosquito-borne encephalitis in the United States (1). It is estimated that upwards of 6

53 million people have been infected by WNV in the US since its introduction in 1999,

54 leading to over a thousand cases of neuroinvasive disease and nearly a hundred deaths

55 each year (2). Following the bite of an infected mosquito, approximately $20 \%$ of

56 individuals present with clinical outcomes ranging from mild febrile illness to severe

57 neuroinvasive disease. Neuroinvasion is a serious complication with long term sequelae

58 that includes ocular involvement, cognitive impairment, muscle weakness, and flaccid

59 paralysis (3). The continued public health threat and lack of FDA-approved vaccines or

60 specific therapeutics against WNV underpins the need to better understand the

61 mechanisms of protective immunity during human infection.

62 The pathogenesis of human WNV infection is incompletely understood, although

63 excellent mouse models have illuminated mechanisms of virus-induced encephalitis and

64 critical features of immune control (4). The bite of an infected mosquito delivers high

65 doses of WNV into the skin where keratinocytes, Langerhans cells, and dermal dendritic

66 cells (DCs) are believed to be initial target cells of infection $(5,6)$. Over the next 24

67 hours, WNV migrates to the skin draining lymph nodes and replicates within resident

68 DCs. Subsequent viremia promotes peripheral seeding of virus into permissive tissues

69 such as the spleen, where DCs are targeted for infection (7). WNV then crosses the

70 blood brain barrier and infects neurons within the central nervous system (CNS),

71 leading to viral encephalitis. Restriction of viral replication by DCs during the early, 
72 peripheral phases of viral replication has been shown to be critical for limiting

73 neuroinvasion and mitigating viral encephalitis $(7,8)$.

74 Within murine DCs, detection of WNV occurs primarily through the concerted

75 efforts of RIG-I and MDA5 $(9,10)$, members of the retinoic acid inducible gene I (RIG-I)

76 like receptor (RLR) family of cytosolic pattern recognition receptors. Signal transduction

77 through the adaptor protein mitochondrial antiviral signaling (MAVS) triggers the nuclear

78 factor-kB (NFKB) and interferon regulatory factor (IRF)-3, 5, and 7 dependent induction

79 of type I interferon (IFN) and antiviral effector gene transcription (8). Following the

80 MAVS-dependent secretion of type I IFN (10), signaling through the type I IFN receptor

81 on DCs is required for early virus restriction and host survival (7).

82 In addition to direct restriction of viral replication, DCs are critical for the

83 programming of antiviral CD8 $+\mathrm{T}$ cell responses that are required for clearance of WNV

84 from the peripheral tissues and CNS (11). In humans, analysis of CD4+ and CD8+ T

85 cells from the blood of WNV-infected patients has found dysfunctional T cell responses

86 correlate with symptomatic disease outcome $(12,13)$. Decreased frequencies of CD4+

87 regulatory $\mathrm{T}$ cells also correlates with symptomatic $\mathrm{WNV}$ infection, highlighting the

88 importance of a balanced T cell response (14). However, the contributions of human

89 DCs in programming $\mathrm{T}$ cell immunity during human WNV infection remains poorly

90 understood.

91 Here, we utilized primary human cells to demonstrate that WNV productively

92 replicates within monocyte-derived DCs. Log-phase viral replication corresponded with

93 induction of type I IFN and antiviral effector genes, with more delayed secretion of IFNa

94 and IFNß proteins. Activation of RLR or type I IFN signaling restricted viral replication, 
95 with RLR signaling remaining effective even after blockade of signaling through the type

96 I IFN receptor. In contrast, WNV infection failed to up-regulate molecules involved in

97 promoting inflammatory responses and priming of $\mathrm{T}$ cell immunity. Functionally,

98 impaired DC activation resulted in diminished $\mathrm{T}$ cell proliferation by WNV-infected

99 moDCs during an allogeneic response. Combined, WNV infection of human DCs

100 activated antiviral responses while failing to program DCs to effectively prime WNV-

101 specific T cell immunity.

\section{Results}

\section{WNV productively infects human DCs}

105 While DCs are an important cell type during infection with multiple flaviviruses,

106 their contributions during human WNV infection remains limited. To model viral

107 replication in human DCs, monocyte-derived DCs (moDCs) were generated from

108 peripheral blood CD14+ monocytes and infected with a pathogenic strain of WNV (15).

109 Viral replication was first detected at $12 \mathrm{hpi}$, as noted by increased viral RNA synthesis

110 (Fig. 1A). Viral RNA levels continued to increase exponentially over the next 36hrs.

111 Consistent with genome replication kinetics, release of infectious virus increased

112 exponentially between 12 and 24hpi and plateaued at 48hpi (Fig. 1B). Next, infected

113 populations of moDCs were stained for intracellular expression of a structural protein

114 found within the virus envelope (viral E protein) (16). Corresponding with log phase viral

115 growth, the percentage of infected cells increased exponentially between 12 and $24 \mathrm{hpi}$

116 (Fig. 1C). Infection plateaued between 24 and $48 \mathrm{hpi}$, reaching upwards of $50 \%$ of cells

117 positive for viral E protein. E protein expression was not observed in mock or UV 
118 inactivated virus infection controls. ImageStream analysis revealed that WNV E protein

119 was localized predominantly within the cytoplasm and did not co-localize with the cell

120 surface marker CD11c or the nucleus (Fig. 1D). Declining percent infection at $72 \mathrm{hpi}$

121 corresponded with significant loss of cell viability (Fig. 1E). Combined, three

122 complementary measures of viral replication (viral RNA, infectious virus release, and

123 viral E protein staining) confirm that human moDCs are productively infected by WNV

124 with log phase viral growth beginning between 12 and $24 \mathrm{hpi}$.

126 Innate immune signaling restricts WNV replication.

127 Type I IFN within dendritic cells is critical for mediating protection against lethal

128 infection outcome and controlling flavivirus replication $(17,18)$. We next determined the

129 ability of both the RLR and type I IFN signaling pathways to restrict WNV infection of

130 moDCs. We infected moDCs with WNV (MOI 10), treated cells with either a RIG-I

131 agonist, MDA5 agonist, or IFN $\beta$ at $1 \mathrm{hpi}$ and virus replication was measured at $24 \mathrm{hpi}$.

132 We triggered RIG-I using a previously characterized and highly-specific agonist derived

133 from the 3' UTR of hepatitis C virus (19) and triggered MDA5 using high molecular

134 weight poly $(\mathrm{I}: \mathrm{C})$, which preferentially activates MDA5 signaling upon delivery into the

135 cytoplasm (20). Stimulation of RIG-I, MDA5, or IFN- $\beta$ signaling potently restricted viral

136 replication, with greater than $90 \%$ inhibition as measured by both viral burden in the

137 supernatant and frequency of infected cells (Fig. 2A). To confirm the role of type I IFN,

138 we infected moDCs in the presence of an IFNAR2 blocking antibody and observed no

139 effect on viral replication through $24 \mathrm{hpi}$, however, late viral control was compromised

140 as shown by a 3-fold increase in the frequency of infected cells and a log-fold increase 
141 in viral replication at 48 hpi (Fig. 2B). Notably, blocking type I IFN signaling in the

142 presence of either RIG-I or MDA5 agonists still reduced viral replication, although we

143 did observe a slight reduction in the efficiency of MDA5 signaling to reduce WNV

144 infection in the presence of an anti-IFNAR2 neutralizing antibody (Fig. 2C) (21)

145 Combined, these findings demonstrate that RIG-I, MDA5 and type I IFN signaling can

146 efficiently block WNV replication in human DCs.

148 WNV induces antiviral and type I IFN responses in human DCs

149 Traditional studies of antiviral responses have predominantly relied on

150 approaches involving genetic ablation, gene knockdown, or gene overexpression

151 methodologies (22). While useful, these approaches remain difficult to perform in

152 primary human cells and may not accurately reflect the role of a given molecule during

153 the normal course of infection. To overcome these limitations, we employed a systems

154 biology approach to assess the antiviral landscape during WNV infection in human DCs

155 (Fig. 3A). We generated moDCs from 5 donors and performed messenger RNA

156 sequencing following innate immune agonist treatment or infection with WNV. To study

157 the early antiviral response during WNV infection, transcriptional responses were

158 measured preceding (12 hpi) and during (24 hpi) log phase viral replication (Fig. 1).

159 Using weighted gene co-expression network analysis (WGCNA), we defined molecular

160 signatures following stimulation of RIG-I, MDA5, or IFNß signaling, identifying six

161 clusters of co-expressed genes, or modules (Fig. 3B) (23). The module with the largest

162 gene membership, module 5 (M5), was enriched for genes associated with the biologic

163 process of "Defense response to virus". Module 6 was also enriched for immune 
164 response related genes, while the remaining four modules were enriched for genes

165 involved in biosynthetic processes, cellular metabolism, and stress responses. Given

166 the large gene number and enrichment for antiviral response pathways, we focused our

167 analyses on the M5 module.

168 We next identified differentially expressed genes (DEGs) within the M5 module

169 for each treatment condition, as compared to time-matched untreated and uninfected

170 cells ( $>2$-fold change, significance of $p<0.01$ ). RIG-I agonist treated cells induced a

171 greater number of M5-related genes as compared to either MDA5 or IFN $\beta$ treated cells.

172 In contrast, gene expression within the M5 module during WNV infection was temporally

173 controlled: minimal gene expression at 12 hpi with more robust gene expression by 24

174 hpi (Fig. 3C). MetaCore pathways enrichment analysis.of the M5 DEGs revealed four

175 significantly enriched pathways including "IFN alpha/beta signaling", "Antiviral activation

176 of interferons", "Innate immune response to RNA virus infection" and "Role of PKR in

177 stress-induced antiviral cell response" (Fig. 3D). The expression patterns of host

178 defense transcription factors, PRR signaling molecules, and antiviral effector genes

179 were largely similar between the RIG-I agonist, poly(I:C), and IFN $\beta$-treated DCs at

180 12hrs post stimulation, suggesting that upregulation of these genes are largely

181 mediated through type I IFN signaling (Fig. 4A). Notably, WNV-infected DCs displayed

182 minimal differentially expressed genes at $12 \mathrm{hpi}$, however at $24 \mathrm{hpi}$ numerous antiviral

183 effectors (e.g. IFIT1, IFIT2, IFIT3, RSAD2, OASL), molecules involved in RNA virus

184 sensing (e.g. DDX58, IFIH1, PKR, TLR3), and the antiviral transcription factor IRF7

185 were significantly up-regulated. Molecules involved in type I IFN signaling were also not

186 induced at 12hpi but showed significant enrichment at 24hpi (Fig. 4B). Despite 
187 enrichment of type I IFN genes at 24hpi, secretion of IFNa and IFNß protein was not

188 detected until 48hpi. The lack of detectable IFNa or IFN $\beta$ protein secretion until 48 hpi in

189 human DCs is consistent with the significant increase in viral replication observed at 48

190 hpi when type I IFN signaling was blocked in WNV-infected DCs with an anti-IFNAR2

191 neutralizing antibody (Fig. 2B). Combined, our data demonstrates that WNV infection of

192 human DCs induces notable antiviral gene expression and that type I IFN signaling

193 plays a role in late, but not early, restriction of viral replication

195 WNV infection fails to promote inflammatory and T cell modulatory cytokine responses

We next assessed the induction of inflammatory cytokine and chemokine responses, an important component of antiviral immunity, DC activation, immune cell

199 recruitment, and T cell priming $(24,25)$. In contrast to type I IFN and antiviral effector 200 responses, WNV infection promoted minimal transcriptional enrichment of multiple

201 cytokines involved in inflammatory cytokine responses (e.g. IL-15, IL-7, and IL-27), and

202 chemotaxis (e.g. CCL2, CCL3, CCL4, CCL5, and CXCL9) (Fig. 5A). CXCR1

203 transcription was also selectively down-regulated during WNV infection. Importantly,

204 RIG-I agonist treatment induced transcriptional expression of multiple inflammatory and 205 T cell modulatory cytokines, confirming the ability of moDCs to mount pro-inflammatory 206 responses upon innate immune stimulation. While RIG-I agonist induced inflammatory 207 cytokines (IL-6 and GM-CSF), T cell promoting cytokines (IL-4, IL-15 and IL-12) and 208 chemokines (CCL3, CCL5 and CXCL9), WNV-infected moDCs displayed little to no 209 induction at the protein level of these cytokines/chemokines at 24 hpi (Fig. 5B). These 
210 findings strongly suggest that WNV blocks the induction of proinflammatory

211 cytokines/chemokines during infection.

214 WNV infection does not induce molecules involved in T cell priming

215 In addition to the secretion of cytokines that modulate $\mathrm{T}$ cell responses, engagement of

216 viral associated molecular patterns increases the surface expression of $\mathrm{T}$ cell co-

217 signaling and MHC molecules on activated DCs (26). At the transcriptional level, WNV

218 infection failed to induce multiple molecules associated with antigen presentation on

219 MHC (HLA-A, ERAP1), LAMP3 (27), proteasome subunits (PSME1, PSMA2, PSMA4,

220 PSMB10), and CD1D (28) (Fig. 6A). WNV also failed to significantly up-regulate genes

221 involved in T cell co-signaling (e.g. CD80, CD86, CD40) and selectively up-regulated

222 expression of galectin-9 (LGALS9), a ligand for the T cell inhibitory receptor TIM3 (12).

223 These findings were biologically validated by flow cytometry, where WNV infection did

224 not up-regulate cell surface levels of CD80, CD86, CD40 or MHC class II proteins within

225 E protein+ cells at 24hpi or 48hpi (Fig. 6B). Notably, high levels of WNV infection (MOI

226 100) still failed to induce expression of costimulatory and MHC class II molecules (Fig.

227 6C). In contrast to WNV infection, RIG-I agonist significantly up-regulated transcription

228 of multiple molecules involved in antigen presentation and $\mathrm{T}$ cell co-signaling,

229 corresponding with increased cell surface expression of CD80, CD86, CD40, and MHC

230 II proteins. Combined, while WNV infection induces type I IFN and antiviral effector

231 responses, WNV-infected DCs are compromised in their ability to induce inflammatory 
232 and chemotactic mediators important for immune activation, as well as antigen

233 presentation and co-stimulatory molecules required for optimal T cell priming.

\section{WNV-infected DCs dampen allogenic T cell proliferation}

238 To determine if the minimal DC activation induced during WNV infection impairs $T$ cell

239 proliferation, we assessed the capacity of WNV-infected moDCs to drive an allogeneic T

240 cell response. Uninfected moDCs induced notable activation of donor mismatched CD4

241 and CD8 T cells in a DC:T cell ratio dependent manner, as indicated by increased

242 expression of the human T cell activation markers CD38 and HLA-DR (29) (Fig. 7A).

243 Allogenic activation of T cells corresponded with proliferation of upwards of $40 \%$ of CD4

244 and CD8 T cells in a DC:T cell ratio dependent manner (Fig. 7B). In contrast, WNV

245 infected moDCs diminished allogeneic CD4 and CD8 T cell activation, corresponding

246 with significantly lower percentages of CD38+ HLA-DR+ and proliferated T cells.

247 Combined, our data suggests that WNV infection induces minimal enrichment of 248 molecules involved in DC activation, resulting in impaired T cell proliferation.

250 Discussion

251 In this study, we combined virologic and immunologic measures with

252 transcriptomic analysis to better understand antiviral responses during WNV infection in

253 primary human DCs. WNV productively infected human moDCs and induced cell death,

254 coinciding with declining viral growth kinetics. RIG-I, MDA5, and IFN $\beta$ signaling potently 
255 restricted viral replication, corresponding with strong activation of antiviral defense

256 response genes. In contrast, there was minimal up-regulation of inflammatory mediators

257 or molecules involved in T cell priming. Functionally, WNV-infected moDCs promoted

258 impaired allogeneic T cell proliferation and activation.

259 Studies in mice have found that RLR and type I IFN signaling are critical for viral

260 restriction and host survival during WNV infection, however, the contributions of innate

261 immune signaling during infection of human cells remains limited $(7,30)$. Here, we

262 demonstrated that RIG-I, MDA5, and IFNß signaling potently restrict WNV replication

263 through induction of strong antiviral gene transcription, suggesting that similar to mice,

264 RLR and type I IFN signaling are important for viral control during human WNV

265 infection. RIG-I and MDA5 agonists also remained efficient in blocking WNV replication

266 independent of type I IFN signaling, consistent with the ability of RLR signaling to

267 induce antiviral gene expression in the absence of the type I IFN receptor in mice (30).

268 Combined, our results confirm the importance of RLR and type I IFN signaling in the

269 induction of antiviral responses and restriction of viral replication within primary human

270 DCs.

271 These findings are similar to previous work, where WNV infection also failed to

272 induce inflammatory cytokine secretion (31). Infection of moDCs with a non-pathogenic

273 WNV isolate, WNV Kunjin, also induced minimal production of IL-12, despite notable

274 up-regulation of both CD86 and CD40 (28). This suggests that an inability to induce

275 inflammatory cytokine responses may be shared among WNV strains, while pathogenic

276 strains have evolved unique mechanisms to subvert antigen presentation and $\mathrm{T}$ cell

277 activation. The failure of WNV to activate human moDCs is also similar to our recent 
278 work with ZIKV (32). In contrast to WNV and ZIKV, infection of moDCs with the yellow

279 fever virus vaccine strain (YFV-17D) up-regulates multiple inflammatory mediators and

280 surface expression of CD80 and CD86 (26). The ability of YFV-17D to induce strong DC

281 activation may reflect the loss of a viral antagonist during the attenuation process,

282 similar to the ability of WNV Kunjin to induce up-regulation of CD86 and CD40 (28).

283 Alternatively, the ability of YFV-17D to induce DC activation may be an inherent

284 property of certain flaviviruses. Indeed, DENV has also been found to activate

285 inflammatory responses and up-regulate co-stimulatory molecules following infection

286 (33, 34). Altogether, our recent studies may suggest that certain neurotropic flaviviruses

287 are particularly adept at subverting DC:T cell signaling.

288 Due to the largely subclinical presentation of WNV infection in humans, 289 understanding genetic correlates of susceptibility and viral restriction remains

290 exceedingly difficult. However, modeling WNV infection in mice lacks the genetic

291 variation seen within outbred human populations. To combat this issue, the

292 collaborative cross (CC) mouse model system, recombinant inbred mice containing

293 genetic diversity from eight founder mouse strains, has been recently developed to

294 study host antiviral responses within a genetically diverse population (35). Using the CC

295 mouse system, one group observed increased regulatory T cells (Tregs) infiltration and

296 no immunopathology in the brains of asymptomatic WNV-infected mice (36). This

297 corroborated well to earlier human studies showing that increased levels of Tregs

298 correlated to improved outcomes during WNV infection (14). These studies have

299 focused largely on observing WNV-specific T and B cell responses to in the CC model

300 system; however, the effects of these diverse polymorphisms on DC function during 
301 WNV infection remains largely untouched. Similar to our current study in human

302 moDCs, transcriptomic analyses from whole spleens and brains of WNV-infected CC

303 mice have also shown differences in antigen presentation, $\mathrm{T}$ cell signaling, and

304 inflammatory cytokine production $(37,38)$. Altogether, the CC mouse system can be

305 utilized in future studies to recapitulate human disease and understand DC responses

306 during WNV infection.

An important observation of our study was that WNV infection did not trigger DC

308 activation, as determined by upregulation of costimulatory protein expression. Through

309 an allogeneic T cell assay, we found that WNV-infected moDCs were less efficient with

310 inducing CD4+ and CD8+ $\mathrm{T}$ cell proliferation as compared to mock-infected moDCs.

311 WNV-specific $T$ cell responses have been detected in both symptomatic and

312 asymptomatic WNV infection in humans (39). However, quality rather than quantity of

313 the CD4+ and CD8+ T cell responses during WNV infection is an important predictor of

314 symptomatic infection outcome. Dysregulated Th1 CD4+ T cell responses were found to

315 strongly correlate with neuroinvasive disease (13). Additionally, decreased numbers of

316 regulatory $T$ cells have also been implicated in symptomatic and neuroinvasive infection

317 in WNV-infected individuals, suggesting that immunomodulation of WNV-specific T cells

318 responses are essential for avoiding immunopathology (14). Lastly, a recent study

319 linked expression of the inhibitory T cell receptor Tim-3 on T cells with progression to

320 symptomatic disease outcome (12). Combined, these findings demonstrate that

321 development of an effective $T$ cell response is critical for modulating infection outcome

322 (symptomatic vs asymptomatic) during WNV infection. Our studies have now

323 determined that WNV interferes with DC activation, through inhibition of costimulatory 
324 molecule expression and pro-inflammatory cytokine production, which can lead to

325 dysregulated T cell responses, immunopathology and excessive neuronal injury.

326 In summary, our systems biology approach defined the antiviral landscape seen

327 during RLR and type I IFN signaling as well as WNV infection in human DCs. Through

328 our study, we observed that WNV can downregulate numerous genes responsible for

329 establishing proper WNV-specific adaptive immune responses in human DCs,

330 negatively affecting proper CD4+ and CD8+ T cell responses. Altogether, our study

331 significantly advances our understanding of how WNV disrupts antiviral immunity during

332 human infection.

\section{Materials and Methods}

335 Ethics statement. Human peripheral blood mononuclear cells (PBMCs) were obtained

336 from de-identified healthy adult blood donors and processed immediately. All individuals

337 who participated in this study provided informed consent in writing in accordance to the

338 protocol approved by the Institutional Review Board of Emory University,

339 IRB\#00045821, entitled "Phlebotomy of healthy adults for the purpose of evaluation and

340 validation of immune response assays".

342 Viruses. WNV stocks were generated from an infectious clone, WNV isolate TX 2002-

$343 \mathrm{HC}$, and passaged once in Vero cells, as previously described (15). WNV stocks were

344 titrated on Vero cells by plaque assay. moDCs were infected with WNV at MOI 10 for

$3451 \mathrm{hr}$ at $37^{\circ} \mathrm{C}$ in cRPMI (without GM-CSF or IL-4). After 1hr, virus was washed off, cells

346 were resuspended in fresh cRPMI, and incubated at $37^{\circ} \mathrm{C}$ for $3-72$ hours. 
348 Cell lines. Vero cells (WHO Reference Cell Banks) were maintained in complete

349 DMEM. Complete DMEM was prepared as follows: DMEM medium (Corning)

350 supplemented with $10 \%$ fetal bovine serum (Optima, Atlanta Biologics), 2mM L-

351 Glutamine (Corning), 1mM HEPES (Corning), 1mM sodium pyruvate (Corning), 1x MEM

352 Non-essential Amino Acids (Corning), and 1x Antibiotics/Antimycotics (Corning).

353 Complete RPMI was prepared as follows: cRPMI; RPMI 1640 medium (Corning)

354 supplemented with $10 \%$ fetal bovine serum (Optima, Atlanta Biologics), 2mM L-

355 Glutamine (Corning), 1mM Sodium Pyruvate (Corning), 1x MEM Non-essential Amino

356 Acids (Corning), and 1x Antibiotics/Antimycotics (Corning).

358 Generation of monocyte derived dendritic cells. To generate human moDCs, CD14+

359 monocytes were differentiated in cRPMI supplemented with $100 \mathrm{ng} / \mathrm{mL}$ of GM-CSF and

360 IL-4 for 5-6 days, as previously described (32). In brief, freshly isolated PBMCs obtained

361 from healthy donor peripheral blood (lymphocyte separation media; StemCell

362 Technologies) were subjected to CD14+ magnetic bead positive selection using the

363 MojoSort Human CD14 Selection Kit (BioLegend). Purified CD14+ monocytes were

364 cultured in complete RPMI supplemented with $100 \mathrm{ng} / \mathrm{mL}$ each of recombinant human

365 IL-4 and GM-CSF (PeproTech) at a cell density of 2 e6 cells $/ \mathrm{mL}$. After $24 \mathrm{hr}$ of culture,

366 media and non-adherent cells were removed and replaced with fresh media and

367 cytokines. Suspension cells ("moDCs") were harvested after 5-6 days of culture and

368 were consistently CD14-, CD11c+, HLA-DR+, DC-SIGN+, and CD1a+ by flow 
369 cytometry. For experimentation, moDCs were maintained in complete RPMI without

370 GM-CSF or IL-4.

372 Quantitative reverse transcription-PCR (qRT-PCR). Total RNA was purified (Quick-

373 RNA MiniPrep Kit; Zymo Research) and viral RNA was reverse transcribed (High

374 Capacity cDNA Kit; Applied Biosystems) using 1 pmol of a GVA tagged (underlined)

375 primer

\section{TTTGCTAGCTTTAGGACCTACTATATCTACCTGGGTCAGCACGTTTGTCATTG-3')}

377 directed against the $\mathrm{E}$ gene $(18,40)$. Reverse transcribed viral sequences were

378 detected by qRT-PCR (TaqMan Gene Expression Master Mix; Applied Biosystems)

379 using 10 pmol of primers (5'-TTTGCTAGCTTTAGGACCTACTATATCTACCT3' and 5'-

380 TCAGCGATCTCTCCACCAAAG-3') and 2.5 pmol of hydrolysis probe (5'-FAM-

381 TGCCCGACCATGGGAGAAGCTC-3IABkFQ-3’). All custom primers and probes were

382 obtained from Integrated DNA Technologies. All qRT-PCR was normalized to the

383 amount of GAPDH (Hs02758991_g1; Applied Biosystems) in each respective sample.

385 Quantitation of infectious virus. Infectious virus was quantitated using a plaque assay

386 on Vero cells with a 1\% agarose overlay and crystal violet counterstain, as previously

387 described (15).

389 Innate immune agonists. To stimulate RIG-I signaling, 100ng of RIG-I agonist derived

390 from the 3'-UTR of hepatitis C virus (19) was transfected per 1e6 cells using TransIT-

391 mRNA transfection kit (Mirus). For stimulation of MDA5 signaling, 100ng of high 
392 molecular weight poly-(I:C) was transfected per 1e6 cells using LyoVec transfection

393 reagent (Invivogen). To stimulate type I IFN signaling, cells were incubated with 100

$394 \mathrm{IU} / \mathrm{mL}$ of human recombinant IFN 3 . In select experiments, different doses of agonists

395 were used and this is indicated within the respective figure legend. To inhibit type I IFN

396 signaling, $5 \mu \mathrm{g} / \mathrm{mL}$ anti-human Interferon- $\alpha / \beta$ Receptor Chain 2 (MMHAR-2; EMD

397 Milipore) blocking monoclonal antibody was used.

398 RNA sequencing and bioinformatics. moDCs were generated from 5 donors and

399 either treated with innate immune agonists for $12 \mathrm{hr}$ (RIG-I, MDA5, or IFN $)$ or infected

400 with WNV (12hpi and 24hpi). Total RNA was purified (Quick-RNA MiniPrep Kit; Zymo

401 Research) and mRNA sequencing libraries were prepared for RNA sequencing (Illumina

402 TruSeq chemistry). RNA sequencing was performed on a Illumina HiSeq 2500 System

403 (100bp single end reads). Sequencing reads were mapped to the human reference

404 genome 38. Reads were normalized and differential expression analysis performed 405 using DESeq2 (41). Differentially expressed genes were determined by 2-fold change 406 and $P<0.01$. The raw data of all RNA sequencing will be deposited into the Gene 407 Expression Omnibus (GEO) repository and the accession number will be available 408 following acceptance of this manuscript. Weighted gene co-expression module analysis 409 was performed on DESeq2 normalized mapped reads (TIBCO Spotfire with Integromics 410 Version 7.0) from RIG-I agonist, MDA5 agonist, IFNß, and mock treated samples. First, 411 the datasets were reduced to focus the network analysis on the 5446 most variable 412 genes (as determined by variation value greater than 1) using the Variance function in

413 R. We constructed a signed weighted correlation network by generating a matrix 414 pairwise correlation between all annotated gene pairs. The resulting biweight mid- 
415 correlation matrix was transformed into an adjacency matrix using the soft thresholding

416 power $(\beta 1)$ of 12 . The adjacency matrix was used to define the topological overlap

417 matrix (TOM) based on a dissimilarity measurement of 1- TO. Genes were hierarchically

418 clustered using average linkage and modules were assigned using the dynamic tree-

419 cutting algorithm (module eigengenes were merged if the pairwise calculation was

420 larger than 0.75$)$. This resulted in the construction of six modules.

422 Flow cytometry. Cells were prepared for analysis as previously described (32). In brief, 423 cells were Fc receptor blocked for $10 \mathrm{~min}$, stained for phenotypic and activation markers

424 for $20 \mathrm{~min}$, and viability stained for $20 \mathrm{~min}$ (Ghost Dye Violet 510, Tonbo Biosciences). For 425 intracellular staining of WNV E protein, cells were fixed and permeabilized (Transcription 426 Factor Staining Buffer Kit, Tonbo Biosciences) and labeled with E16-APC for 20min at room 427 temperature (16). Flow cytometry data was analyzed using FlowJo version 10 software. 428 ImageStream data was analyzed using the Amnis IDEAS software. For moDC studies, the 429 following antibody clones from Biolegend were used: CD11c (B-Ly6), CD80 (2D10), CD86 430 (IT2.2), CD40 (5C3), HLA-DR (G46-6; BD Bioscience), CD14 (M5E2), CD1a (HI149).

431 T cell proliferation assay. Freshly isolated PBMCs obtained from healthy donor 432 peripheral blood (lymphocyte separation media; StemCell Technologies) were subjected 433 to CD4 or CD8 T cell magnetic bead negative selection using the MojoSort Human CD4 434 or CD8 Selection Kit (BioLegend). Isolated CD4 or CD8 T cells were labeled with 435 CellTrace Violet (CTV) Cell Proliferation Kit (ThermoFisher) per the manufacturer's 436 instructions. In a 96-well U bottom plate, CTV labeled CD4 or CD8 T cells (2e5 ells) 437 were mixed with different ratios of either uninfected moDCs, or moDCs infected with 
438 WNV for $24 \mathrm{hr}(1: 4,1: 8,1: 16,1: 32,1: 64$, and 1:128 DC:T cell ratios). To prevent

439 spreading infection, we added anti-E16 neutralizing antibody at $5 \mu \mathrm{g} / \mathrm{mL}$ throughout the

440 DC:T cell co-culture period (16). After 6 days of co-culture, cells were stained for

441 surface expression of CD4 or CD8, CD3, CD38, and HLA-DR. Proliferation, by CTV

442 dilution, and T cell activation (CD38+HLA-DR+) were assessed by flow cytometry (42).

444 Multiplex bead array. Cytokine analysis was performed on supernatants using a

445 human 25-plex panel (ThermoScientific) and a custom 2-plex panel with human IFN $\beta$

446 and IFNa simplex kits (eBioscience) as described previously (32). Cytokines analyzed

447 included: IFN- $\alpha$, IFN $\beta$, GM-CSF, TNF- $\alpha$, IL-4, IL-6, MIP-1 $\alpha$, IL-8, IL-15, IL-2R, IP-10,

448 MIP-1 $\beta$, Eotaxin, RANTES, MIG, IL-1RA, IL-12 (p40/p70) IL-13, IFN- $\gamma$, MCP-1, IL-7, IL-

449 17, IL-10, IL-5, IL-2, and IL-1 $\beta$.

450

451 Statistics. All statistical analysis was performed using GraphPad Prism version 6

452 software. The number of donors varied by experiment and is indicated within the figure

453 legends. Statistical significance was determined as $\mathrm{P}<0.05$ using a Kruskal-Wallis test

454 (when comparing more than two groups lacking paired measurements), a Wilcoxon test

455 (when comparing two groups with paired measurements), or a two-way ANOVA (when

456 comparing two groups across multiple independent variables). All comparisons were

457 made between treatment or infection conditions with a time point matched, uninfected

458 and untreated control. 


\section{Funding Information.}

460 This work was funded in part by National Institutes of Health grants U19AI083019

461 (M.S.S), R56Al110516 (M.S.S) and R21Al113485 (M.S.S.), 2U19AI090023 (B.P),

462 5R37DK057665 $\quad$ (B.P), 5R37AI048638 $\quad$ (B.P), 2U19AI057266 $\quad$ (B.P), ORIP/OD

463 P51OD011132 (M.S.S, B.P), Emory University Department of Pediatrics Junior Faculty

464 Focused Award (M.S.S), Children's Healthcare of Atlanta, Emory Vaccine Center, and

465 The Georgia Research Alliance (M.S.S). The funders had no role in study design, data

466 collection and analysis, decision to publish, or preparation of the manuscript. 


\section{Acknowledgements.}

468 We thank the Children's Healthcare of Atlanta and Emory University Pediatric Flow

469 Cytometry Core for providing access to flow cytometry, ImageStream, and Luminex

470 systems, and the Yerkes Genomics Core for performing RNA sequencing. 


\section{References}

472 1. Lindsey NP, Lehman JA, Staples JE, Fischer M. 2015. West Nile Virus and Other

473 Nationally Notifiable Arboviral Diseases - United States, 2014. MMWR Morb Mortal

474

475 Wkly Rep 64:929-34.

476

477

2. Chancey C, Grinev A, Volkova E, Rios M. 2015. The global ecology and epidemiology of West Nile virus. Biomed Res Int 2015:376230.

478

479

3. Patel H, Sander B, Nelder MP. 2015. Long-term sequelae of West Nile virus-related
illness: a systematic review. Lancet Infect Dis 15:951-9.

4. Suthar MS, Diamond MS, Gale M, Jr. 2013. West Nile virus infection and immunity. Nat Rev Microbiol 11:115-28.

5. Schmid MA, Harris E. 2014. Monocyte recruitment to the dermis and differentiation to dendritic cells increases the targets for dengue virus replication. PLoS Pathog 10:e1004541.

483

6. Lim PY, Behr MJ, Chadwick CM, Shi PY, Bernard KA. 2011. Keratinocytes are cell targets of West Nile virus in vivo. J Virol 85:5197-201.

7. Pinto AK, Ramos HJ, Wu X, Aggarwal S, Shrestha B, Gorman M, Kim KY, Suthar MS, Atkinson JP, Gale M, Jr., Diamond MS. 2014. Deficient IFN signaling by myeloid cells leads to MAVS-dependent virus-induced sepsis. PLoS Pathog 10:e1004086.

8. Lazear HM, Lancaster A, Wilkins C, Suthar MS, Huang A, Vick SC, Clepper L, Thackray L, Brassil MM, Virgin HW, Nikolich-Zugich J, Moses AV, Gale M, Jr., Fruh K, Diamond MS. 2013. IRF-3, IRF-5, and IRF-7 coordinately regulate the type I IFN response in myeloid dendritic cells downstream of MAVS signaling. PLoS Pathog 9:e1003118.

9. Errett JS, Suthar MS, McMillan A, Diamond MS, Gale M, Jr. 2013. The essential, nonredundant roles of RIG-I and MDA5 in detecting and controlling West Nile virus infection. J Virol 87:11416-25.

10. Suthar MS, Ma DY, Thomas S, Lund JM, Zhang N, Daffis S, Rudensky AY, Bevan MJ, Clark EA, Kaja MK, Diamond MS, Gale M, Jr. 2010. IPS-1 is essential for the control of West Nile virus infection and immunity. PLoS Pathog 6:e1000757.

500

501

502

503

504

505

506

507

508

509

510

511

11. Hildner K, Edelson BT, Purtha WE, Diamond M, Matsushita H, Kohyama M, Calderon B, Schraml BU, Unanue ER, Diamond MS, Schreiber RD, Murphy TL, Murphy KM. 2008. Batf3 deficiency reveals a critical role for CD8alpha+ dendritic cells in cytotoxic T cell immunity. Science 322:1097-100.

12. Lanteri MC, Diamond MS, Law JP, Chew GM, Wu S, Inglis HC, Wong D, Busch MP, Norris PJ, Ndhlovu LC. 2014. Increased frequency of Tim-3 expressing T cells is associated with symptomatic West Nile virus infection. PLoS One 9:e92134.

13. James EA, Gates TJ, LaFond RE, Yamamoto S, Ni C, Mai D, Gersuk VH, O'Brien K, Nguyen QA, Zeitner B, Lanteri MC, Norris PJ, Chaussabel D, Malhotra U, Kwok WW. 2016. Neuroinvasive West Nile Infection Elicits Elevated and Atypically Polarized T Cell Responses That Promote a Pathogenic Outcome. PLoS Pathog 12:e1005375.

14. Lanteri MC, O'Brien KM, Purtha WE, Cameron MJ, Lund JM, Owen RE, Heitman JW, Custer B, Hirschkorn DF, Tobler LH, Kiely N, Prince HE, Ndhlovu LC, Nixon DF, Kamel HT, Kelvin DJ, Busch MP, Rudensky AY, Diamond MS, Norris PJ. 2009. Tregs control the development of symptomatic West Nile virus infection in humans and mice. J Clin Invest 119:3266-77. 
15. Suthar MS, Brassil MM, Blahnik G, Gale M, Jr. 2012. Infectious clones of novel lineage 1 and lineage 2 West Nile virus strains WNV-TX02 and WNV-Madagascar. J Virol 86:7704-9.

16. Oliphant T, Engle M, Nybakken GE, Doane $C$, Johnson S, Huang L, Gorlatov S, Mehlhop E, Marri A, Chung KM, Ebel GD, Kramer LD, Fremont DH, Diamond MS. 2005. Development of a humanized monoclonal antibody with therapeutic potential against West Nile virus. Nat Med 11:522-30.

17. Keller BC, Fredericksen BL, Samuel MA, Mock RE, Mason PW, Diamond MS, Gale M, Jr. 2006. Resistance to alpha/beta interferon is a determinant of West Nile virus replication fitness and virulence. J Virol 80:9424-34.

18. Samuel MA, Diamond MS. 2005. Alpha/beta interferon protects against lethal West Nile virus infection by restricting cellular tropism and enhancing neuronal survival. J Virol 79:13350-61.

19. Saito T, Owen DM, Jiang F, Marcotrigiano J, Gale M, Jr. 2008. Innate immunity induced by composition-dependent RIG-I recognition of hepatitis C virus RNA. Nature 454:523-7.

20. Kato H, Takeuchi 0, Mikamo-Satoh E, Hirai R, Kawai T, Matsushita K, Hiiragi A, Dermody TS, Fujita T, Akira S. 2008. Length-dependent recognition of doublestranded ribonucleic acids by retinoic acid-inducible gene-I and melanoma differentiation-associated gene 5. J Exp Med 205:1601-10.

21. Kim YE, Ahn JH. 2015. Positive role of promyelocytic leukemia protein in type I interferon response and its regulation by human cytomegalovirus. PLoS Pathog 11:e1004785.

22. Bowen JR, Ferris MT, Suthar MS. 2016. Systems biology: A tool for charting the antiviral landscape. Virus Res 218:2-9.

540 23. Langfelder P, Horvath S. 2008. WGCNA: an R package for weighted correlation

541

542

543

544

545

546 network analysis. BMC Bioinformatics 9:559.

24. Seder RA, Paul WE, Davis MM, Fazekas de St Groth B. 1992. The presence of interleukin 4 during in vitro priming determines the lymphokine-producing potential of CD4+ T cells from T cell receptor transgenic mice. J Exp Med 176:10918.

25. Heufler C, Koch F, Stanzl U, Topar G, Wysocka M, Trinchieri G, Enk A, Steinman RM, mediates $\mathrm{T}$ helper 1 development as well as interferon-gamma production by $\mathrm{T}$ helper 1 cells. Eur J Immunol 26:659-68.

26. Querec T, Bennouna S, Alkan S, Laouar Y, Gorden K, Flavell R, Akira S, Ahmed R, Pulendran B. 2006. Yellow fever vaccine YF-17D activates multiple dendritic cell subsets via TLR2, 7, 8, and 9 to stimulate polyvalent immunity. J Exp Med 203:41324.

27. de Saint-Vis B, Vincent J, Vandenabeele S, Vanbervliet B, Pin JJ, Aït-Yahia S, Patel S, Mattei MG, Banchereau J, Zurawski S, Davoust J, Caux C, Lebecque S. 1998. A Novel Lysosome-Associated Membrane Glycoprotein, DC-LAMP, Induced upon DC Maturation, Is Transiently Expressed in MHC Class II Compartment. Immunity 9:325-336. 
28. Kovats S, Turner S, Simmons A, Powe T, Chakravarty E, Alberola-Ila J. 2016. West Nile virus-infected human dendritic cells fail to fully activate invariant natural killer T cells. Clin Exp Immunol 186:214-226.

29. Akondy RS, Monson ND, Miller JD, Edupuganti S, Teuwen D, Wu H, Quyyumi F, Garg S, Altman JD, Del Rio C, Keyserling HL, Ploss A, Rice CM, Orenstein WA, Mulligan MJ, Ahmed R. 2009. The yellow fever virus vaccine induces a broad and polyfunctional human memory CD8+ T cell response. J Immunol 183:7919-30.

30. Suthar MS, Brassil MM, Blahnik G, McMillan A, Ramos HJ, Proll SC, Belisle SE, Katze MG, Gale M, Jr. 2013. A systems biology approach reveals that tissue tropism to West Nile virus is regulated by antiviral genes and innate immune cellular processes. PLoS Pathog 9:e1003168.

31. Silva MC, Guerrero-Plata A, Gilfoy FD, Garofalo RP, Mason PW. 2007. Differential activation of human monocyte-derived and plasmacytoid dendritic cells by West Nile virus generated in different host cells. J Virol 81:13640-8.

32. Bowen JR, Quicke KM, Maddur MS, O'Neal JT, McDonald CE, Fedorova NB, Puri V, Shabman RS, Pulendran B, Suthar MS. 2017. Zika Virus Antagonizes Type I Interferon Responses during Infection of Human Dendritic Cells. PLOS Pathogens 13:e1006164.

33. Rodriguez-Madoz JR, Bernal-Rubio D, Kaminski D, Boyd K, Fernandez-Sesma A. 2010. Dengue virus inhibits the production of type I interferon in primary human dendritic cells. J Virol 84:4845-50.

34. Olagnier D, Peri S, Steel C, van Montfoort N, Chiang C, Beljanski V, Slifker M, He Z, Nichols CN, Lin R, Balachandran S, Hiscott J. 2014. Cellular oxidative stress response controls the antiviral and apoptotic programs in dengue virus-infected dendritic cells. PLoS Pathog 10:e1004566.

35. Ferris MT, Aylor DL, Bottomly D, Whitmore AC, Aicher LD, Bell TA, BradelTretheway B, Bryan JT, Buus RJ, Gralinski LE, Haagmans BL, McMillan L, Miller DR, Rosenzweig E, Valdar W, Wang J, Churchill GA, Threadgill DW, McWeeney SK, Katze MG, Pardo-Manuel de Villena F, Baric RS, Heise MT. 2013. Modeling host genetic regulation of influenza pathogenesis in the collaborative cross. PLoS Pathog 9:e1003196.

36. Graham JB, Thomas S, Swarts J, McMillan AA, Ferris MT, Suthar MS, Treuting PM, Ireton R, Gale M, Jr., Lund JM. 2015. Genetic diversity in the collaborative cross model recapitulates human West Nile virus disease outcomes. MBio 6:e00493-15.

37. Green R, Wilkins C, Thomas S, Sekine A, Ireton RC, Ferris MT, Hendrick DM, Voss K, de Villena FP, Baric R, Heise M, Gale M, Jr. 2016. Identifying protective host gene expression signatures within the spleen during West Nile virus infection in the collaborative cross model. Genom Data 10:114-117.

38. Green R, Wilkins C, Thomas S, Sekine A, Ireton RC, Ferris MT, Hendrick DM, Voss K, Pardo-Manuel de Villena F, Baric RS, Heise MT, Gale M, Jr. 2016. Transcriptional profiles of WNV neurovirulence in a genetically diverse Collaborative Cross population. Genom Data 10:137-140.

39. Lanteri MC, Heitman JW, Owen RE, Busch T, Gefter N, Kiely N, Kamel HT, Tobler LH, Busch MP, Norris PJ. 2008. Comprehensive analysis of west nile virus-specific T cell responses in humans. J Infect Dis 197:1296-306. 
604 40. Lim SM, Koraka P, Osterhaus AD, Martina BE. 2013. Development of a strand605 specific real-time qRT-PCR for the accurate detection and quantitation of West Nile 606 virus RNA. J Virol Methods 194:146-53.

607 41. Love MI, Huber W, Anders S. 2014. Moderated estimation of fold change and 608 dispersion for RNA-seq data with DESeq2. Genome Biol 15:550.

42. McElroy AK, Akondy RS, Davis CW, Ellebedy AH, Mehta AK, Kraft CS, Lyon GM, Ribner BS, Varkey J, Sidney J, Sette A, Campbell S, Stroher U, Damon I, Nichol ST, Spiropoulou CF, Ahmed R. 2015. Human Ebola virus infection results in substantial immune activation. Proc Natl Acad Sci U S A 112:4719-24. 


\section{Figure Captions}

615 Fig 1. WNV productively infects human moDCs. moDCs were infected with WNV or

616 UV-inactivated WNV (UV-WNV) at MOI 10 (as determined on Vero cells) and analyzed

617 at indicated hours post-infection. (A) Viral RNA as quantitated in cell lysates by RT-

618 qPCR. Shown as $\log _{2}$ normalized expression after normalization to GAPDH. Data is

619 shown for each donor with the mean ( $n=5-11$ donors). (B) Infectious virus release into

620 the supernatant as determined by a viral plaque assay on Vero cells. Data is shown for

621 each donor with the mean ( $n=4-17$ donors). PFU, plaque-forming unit. (C) Percent $E$

622 protein+ cells as determine by flow cytometry (Left panel). Data is shown for each donor

623 with the mean ( $n=5-31$ donors). (D) ImageStream analysis of WNV-infected moDCs

624 labeled for viral E protein at 24hpi (E) Percent viable cells. Data is shown for each donor

625 with the mean ( $\mathrm{n}=5$ donors).

627 Fig 2. Innate immune signaling restricts WNV replication. (A) Experimental

628 overview. moDCs were infected with WNV at MOI 10 (as determined on Vero cells) for

$6291 \mathrm{hr}$ and then treated with RIG-I agonist (100ng/1e6 cells), MDA5 agonist (100ng/1e6

630 cells), IFNß (1000 IU/mL), or left untreated ("WNV"). (B) Infectious virus release into the

631 supernatant (left panel) or viral E protein staining (right panel) was assessed at $24 \mathrm{hpi}$.

632 Data is represented as percent inhibition and shown for each donor with the mean ( $\mathrm{n}=$

633 5-6 donors). (C) moDCs treated as in B were incubated with or without anti-IFNAR2

$634(5 \mu \mathrm{g} / \mathrm{mL})$. For $\mathrm{A}$ and $\mathrm{C}$, percent inhibition was calculated as: (1 - [WNV + agonist] /

635 [WNV alone]) * 100. Dashed line indicates $100 \%$ inhibition, or complete block of viral 
636 infection. Data is shown for each donor with the mean ( $n=3$ donors). Statistical

637 significance was determined as $\mathrm{P}<0.05$ using a Kruskal-Wallis test.

639 Fig 3. (A) Overview of systems biology approach used in this study. (B) Topologic 640 overlap matrix showing enriched modules defined by WGCNA following $12 \mathrm{hr}$ treatment 641 with RIG-I agonist (100ng/1e6 cells), MDA5 agonist (100ng/1e6 cells), or IFN $\beta$ (1000 $642 \mathrm{IU} / \mathrm{mL}$ ). Functional annotation was performed using the DAVID Bioinformatics Resource

643 version 6.8, with the top enriched biological process shown. (C) Heatmap of all module 6445 differentially expressed genes with the $\log _{2}$ normalized fold change relative to

645 uninfected, untreated cells shown. Genes that did not reach the significance threshold 646 are depicted in black color. (D) Top enriched MetaCore canonical pathways of module 5 647 differentially expressed genes relative to uninfected and untreated cells ( $>2$-fold change, $648 \mathrm{p}<0.01)$. Node size corresponds with the pathway enrichment significance score $\left(-\log _{10}\right.$ 649 p value) for each indicated treatment condition.

651 Fig 4. WNV induces robust antiviral and type I IFN responses. Messenger RNA 652 sequencing was performed on moDCs generated from 5 donors after RIG-I agonist 653 (100ng/1e6 cells for $12 \mathrm{hrs})$, high MW poly(l:C) MDA5 agonist (100 ng/1e6 cells), or 654 IFNß (100 IU/mL) treatment or WNV infection (MOI 10; 12 and 24hpi). (A) Heatmap of 655 differentially expressed genes (DEGs) corresponding to antiviral transcription factors, 656 innate immune sensors, and antiviral effector genes. Genes that did not reach the 657 significance threshold are depicted in black color. (B) Heatmap of DEGs corresponding 658 to type I IFN responses. For all heatmaps, the $\log _{2}$ normalized fold change relative to 
659 uninfected, untreated cells is shown ( $>2$-fold change, significance of $p<0.01$ ). Genes

660 that did not reach the significance threshold are depicted in black color. Each column

661 within a treatment condition is marked by a unique color and represents a different

662 donor ( $n=5$ donors). (C) Secretion of IFNa and IFN $\beta$ proteins into the supernatant

663 following RIG-I agonist treatment (100ng/1e6 cells), infection with UV-inactivated WNV

664 (MOI 10, "UV-WNV"), or infection with replication competent WNV (MOI 10, "WNV").

665 Data is shown for each donor with the mean ( $n=4-11$ donors). Statistical significance

666 was determined as $\mathrm{P}<0.05$ using a Kruskal-Wallis test.

668 Fig 5. WNV infected DCs do not generate robust proinflammatory cytokine and

669 chemokine responses. (A) Heatmap of genes involved in inflammatory cytokine

670 responses and chemotaxis. The $\log _{2}$ normalized fold change relative to uninfected,

671 untreated cells is shown ( $>2$-fold change, significance of $p<0.01$ ). Genes that did not

672 reach the significance threshold are depicted in black color. Each column within a

673 treatment condition is marked by a unique color and represents a different donor $(n=5$

674 donors). (B) Secretion of inflammatory cytokines, $T$ cell modulatory cytokines, and

675 chemokines were assessed by multiplex bead array following RIG-I agonist treatment

676 (100ng/1e6 cells), infection with UV-inactivated WNV (MOI 10, "UV-WNV"), or infection

677 with replication competent WNV (MOI 10, "WNV"). Responses were assessed at 24hr

678 following treatment or infection. Data for each donor is shown with the mean $(n=4-7$

679 donors). Statistical significance was determined as $\mathrm{P}<0.05$ using a Kruskal-Wallis test. 


\section{Fig 6. WNV-infected DCs fail to increase expression of molecules involved in}

682 antigen presentation and $\mathbf{T}$ cell co-stimulation. (A) Heatmap of genes involved in

683 antigen processing and presentation or $\mathrm{T}$ cell co-signaling. The $\log _{2}$ normalized fold 684 change relative to uninfected, untreated cells is shown ( $>2$-fold change, significance of $685 \mathrm{p}<0.01)$. Genes that did not reach the significance threshold are depicted in black color. 686 Each column within a treatment condition is marked by a unique color and represents a 687 different donor ( $n=5$ donors). (B) Cell surface expression of CD80, CD86, CD40, or $688 \mathrm{MHC}$ II was quantitated by flow cytometry following RIG-I agonist treatment (100ng/1e6 689 cells), infection with UV-inactivated WNV (MOI 10, "UV-WNV"), or infection with 690 replication competent WNV (MOI 10, "WNV”). Responses were assessed at 24hr and 691 48hr following treatment or infection. (C) Cell surface expression of CD80, CD86, CD40, 692 or MHC II was quantitated by flow cytometry following infection with increasing MOls of 693 WNV at 24 hpi (MOI 0.1, 1, 10, and 100). For (B) and (C), WNV-infected moDCs were 694 labeled for viral $E$ protein and data is shown for the $E$ protein+ population. Data for each 695 donor is shown as median fluorescence intensity (MFI) with the mean (n=3-5 donors). 696 Statistical significance was determined as $P<0.05$ using a Kruskal-Wallis test.

698 Fig 7. WNV-infected DCs are compromised in T cell proliferation. moDCs were left 699 uninfected or infected with WNV (MOI 10) for 24hrs. Allogeneic CD4 or CD8 T cells 700 were labeled with CellTrace violet (CTV) and incubated with uninfected or WNV infected

701 moDCs at the indicated DC:T cell ratios in the presence of an anti-E16 WNV blocking 702 antibody to limit spreading infection $(5 \mu \mathrm{g} / \mathrm{mL})$ for 6 days. (A) The percentage of cells 703 double positive for the T cell activation markers CD38 and HLA-DR on day 6 of 
704 allogeneic co-culture. (B) The percentage of cells that had proliferated by day 6 of

705 allogeneic co-culture. Percent proliferation was defined as any cell that diluted CTV as

706 compared to a "no DC, T cell only control". Statistical significance was determined as P

$707<0.05$ using a two-way ANOVA analysis.

708

709 
Ein 1 robianxiv preprint doi: https://doi.org/10.1101/602839; this version posted April 9, 2019. The copyright holder for this preprint (which was not certified by peer review) is the author/funder. All rights reserved. No reuse allowed without permission.

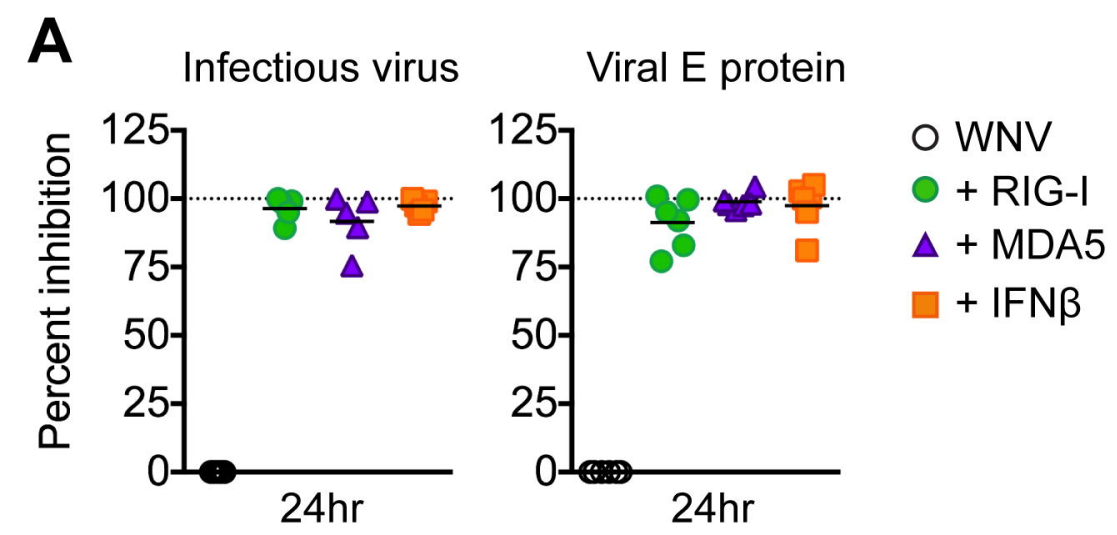

Hours post-infection

B

Infectious virus

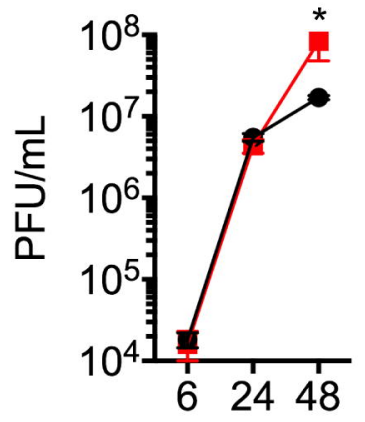

Viral E protein

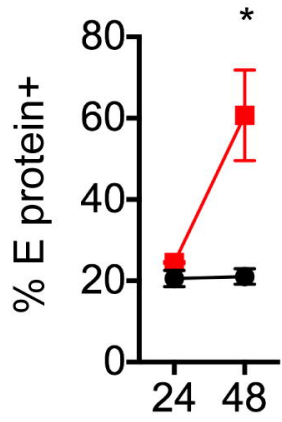

Hours post-infection
C

Viral E protein
O WNV

+ RIG-I

O + RIG-I, + aIFNAR2

$\Delta+$ MDA5

$\Delta+$ MDA5, + alFNAR2

Agonist -++++ alFNAR2 - - + - + 
(1) $\mathrm{CD} 14+\mathrm{moDCs}$

Donor 1

Donor 2

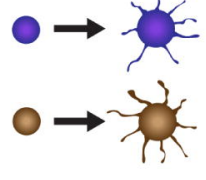

Donor 3

Donor 4

Donor 5

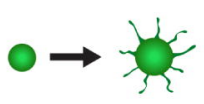

\section{(1)}

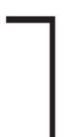

Mock



2

RIG-I agonist

MDA5 agonist

IFN $\beta$

WNV infection

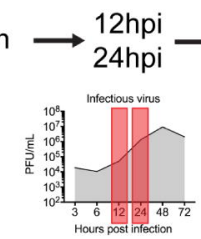

(3) Define antiviral modules using WGCNA of innate immune agonist treatment

4 Use genes within antiviral modules as a filter to assess gene expression during WNV infection

(5) Indentify unique aspects of antiviral immunity during human WNV infection of DCs
5

1. Identify differentially expressed genes (DESeq2.0)

2. Predict transcription factor regulatory networks (iRegulon)

3. Pathway analysis (MetaCore)

(3)

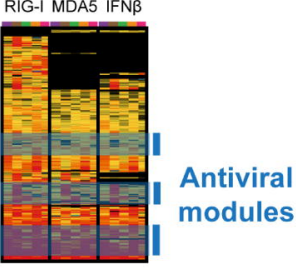

1. Identify which genes are induced by WNV infection

2. Discover viral antagonism of antiviral immune response

Biologically validate findings (Cytokine bead arrays, flow cytometry, western blot)

B

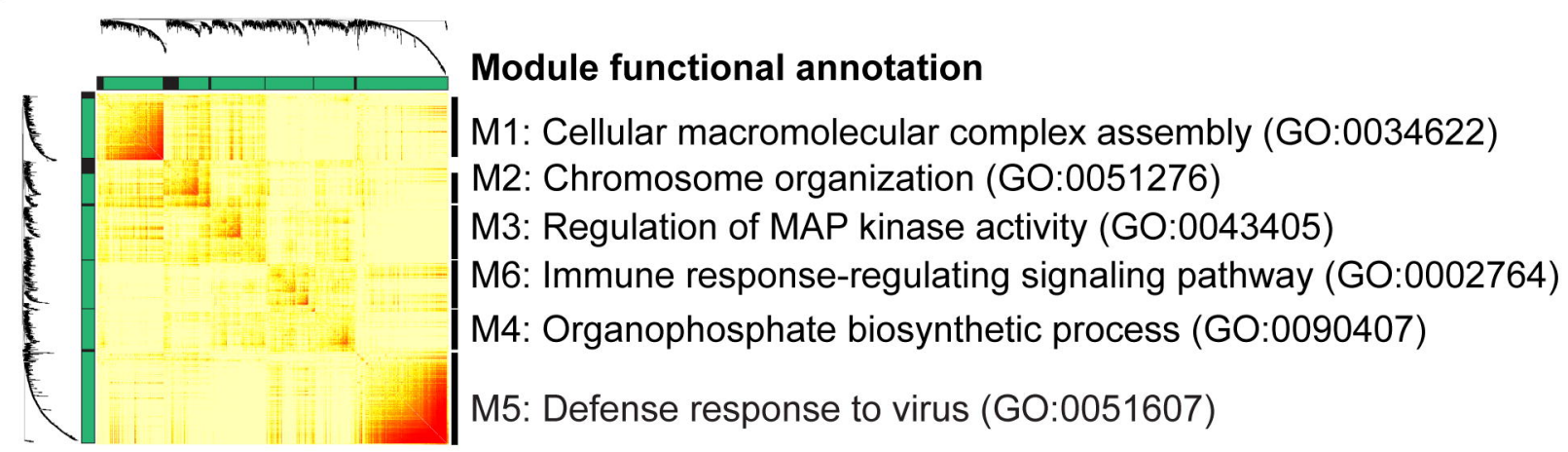

C

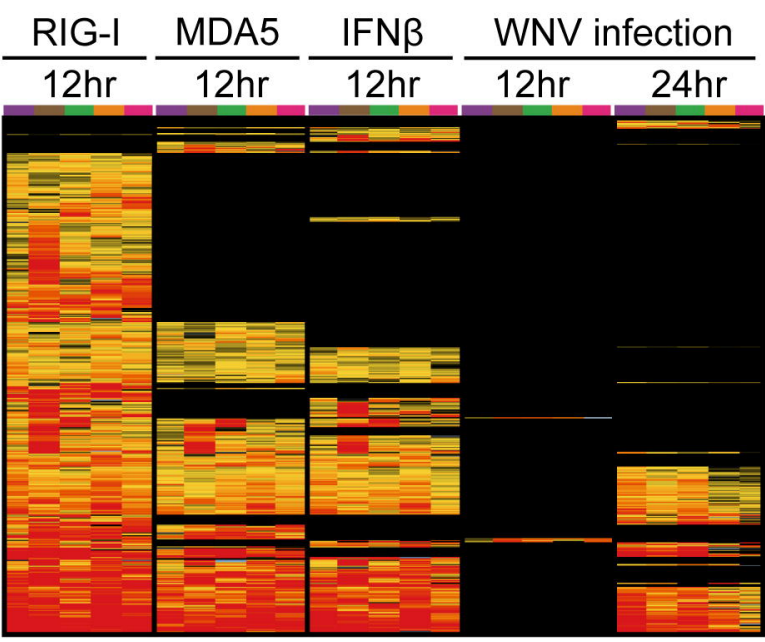

D

\section{MetaCore Canonical Pathway}

1. IFN alpha/beta signaling pathway

2. Antiviral actions of Interferons

3. Innate immune response to RNA viral infection

4. Role of PKR in stress-induced antiviral cell response

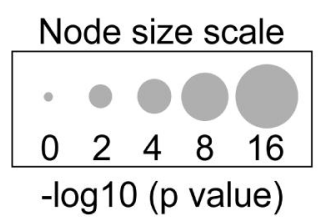

Fold change relative to mock $\left(\log _{2}\right)$ 
bioßxiv preprint doi: https://doi.org/10.1101/602839; this version posted April 9, 2019. The copyright holder for this preprint (which was not certified by peer review) is the author/funder. All rights reserved. No reuse allowed without permission.

Transcription Factors

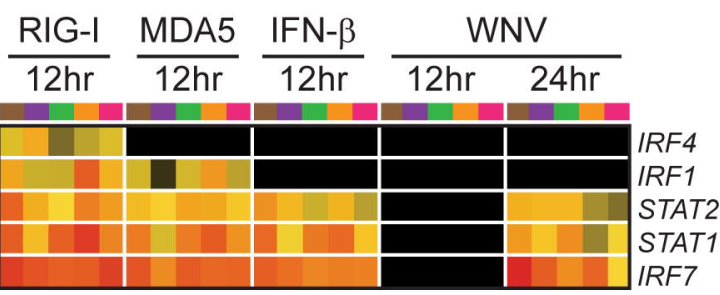

\section{PRR Signaling}
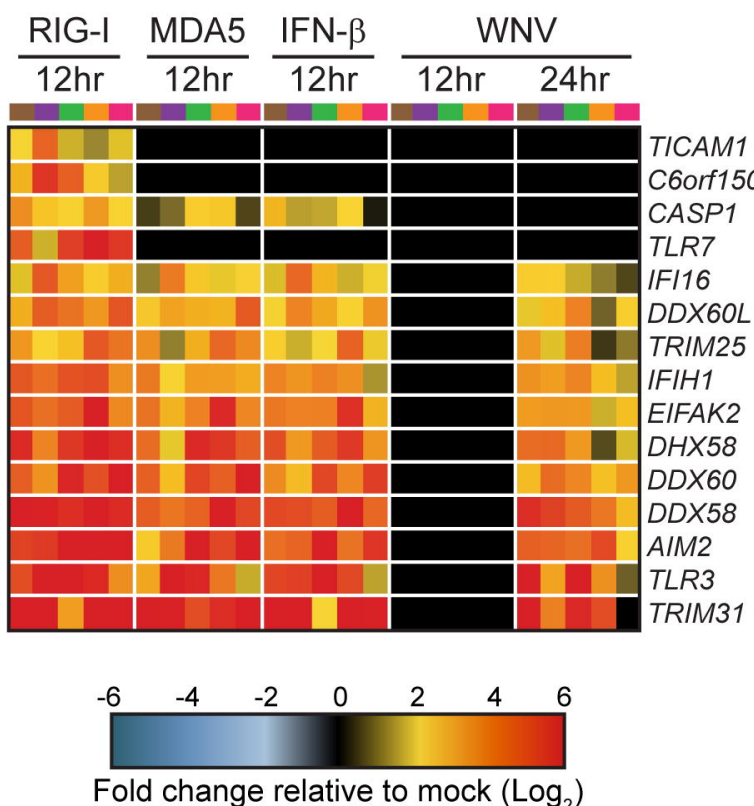

Fold change relative to mock $\left(\log _{2}\right)$

\section{Type I IFN}

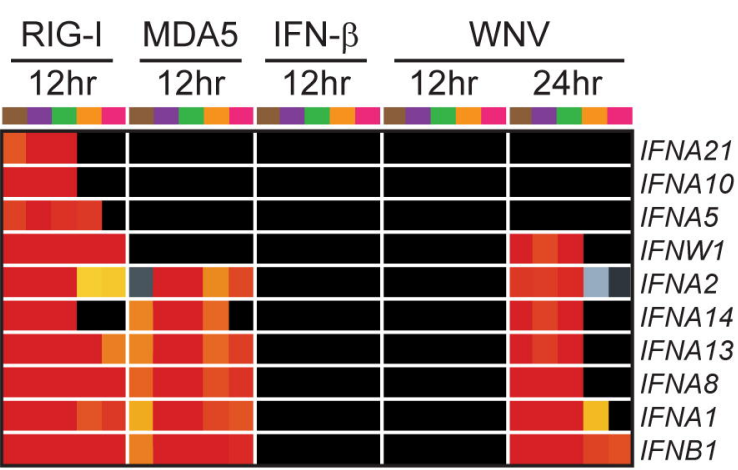

\section{Antiviral Effectors}

$\frac{\mathrm{RIG-I}}{12 \mathrm{hr}} \frac{\mathrm{MDA} 5}{12 \mathrm{hr}} \frac{\mathrm{IFN}-\beta}{12 \mathrm{hr}} \frac{\mathrm{WNV}}{12 \mathrm{hr} 24 \mathrm{hr}} \quad \begin{aligned} & \text { Donor } 2 \\ & \text { Donor 3 } \\ & \text { Donor } 4 \\ & \text { Donor } 5\end{aligned}$

C

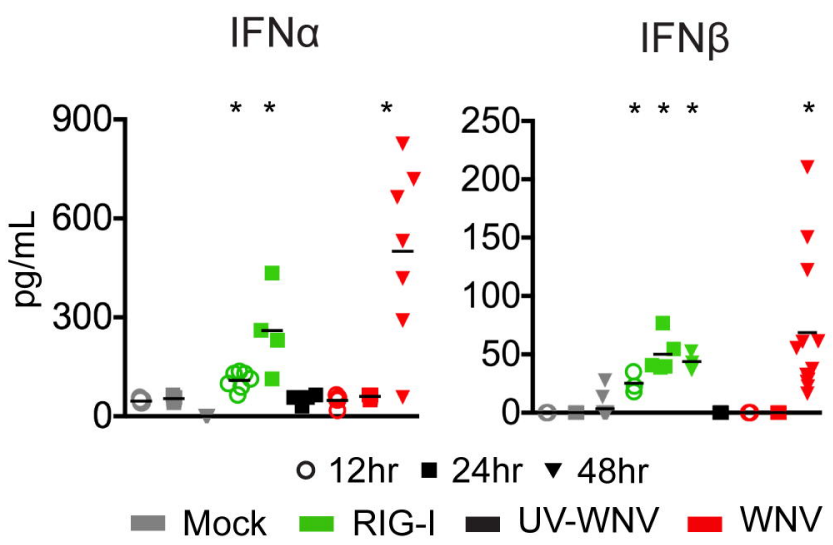


bioßxiv preprint doi: https://doi.org/10.1101/602839; this version posted April 9, 2019. The copyright holder for this preprint (which was not certified by peer review) is the author/funder. All rights reserved. No reuse allowed without permission.
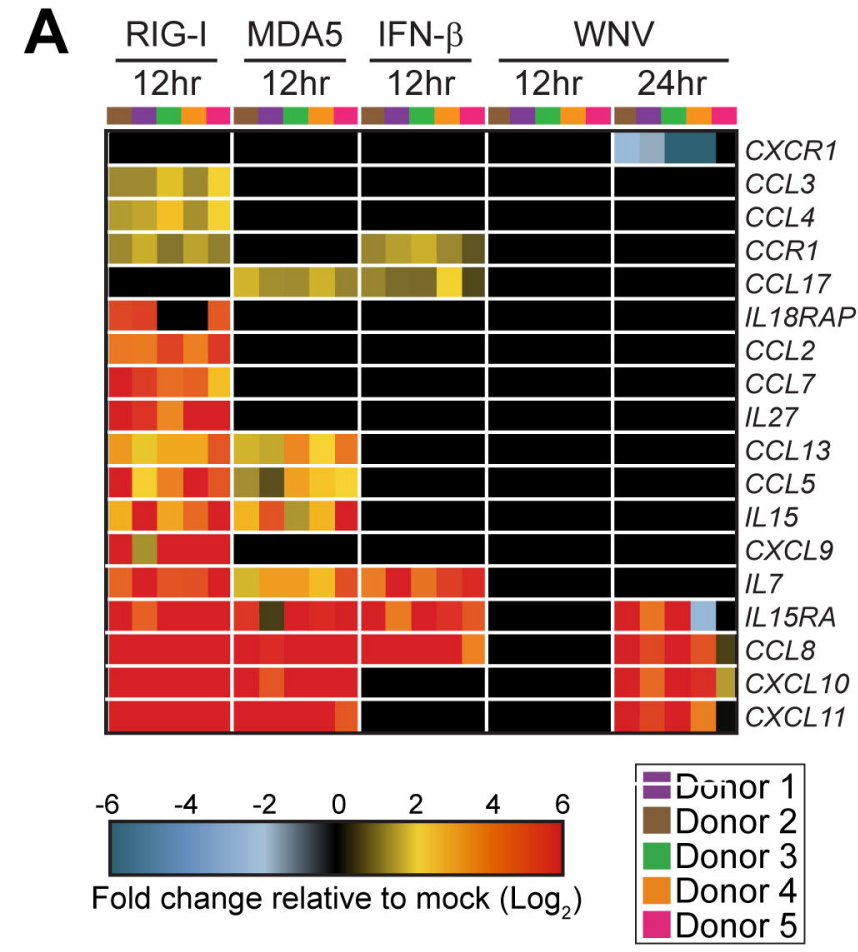

B

MIG (CXCL9) IP-10 (CXCL10)
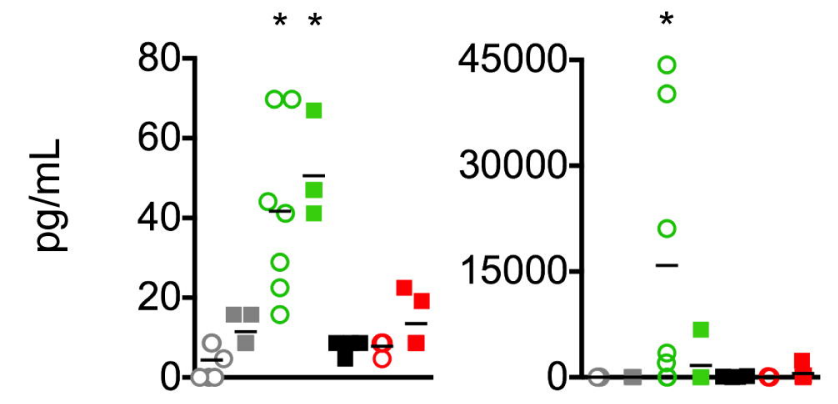

IL-4

GM-CSF
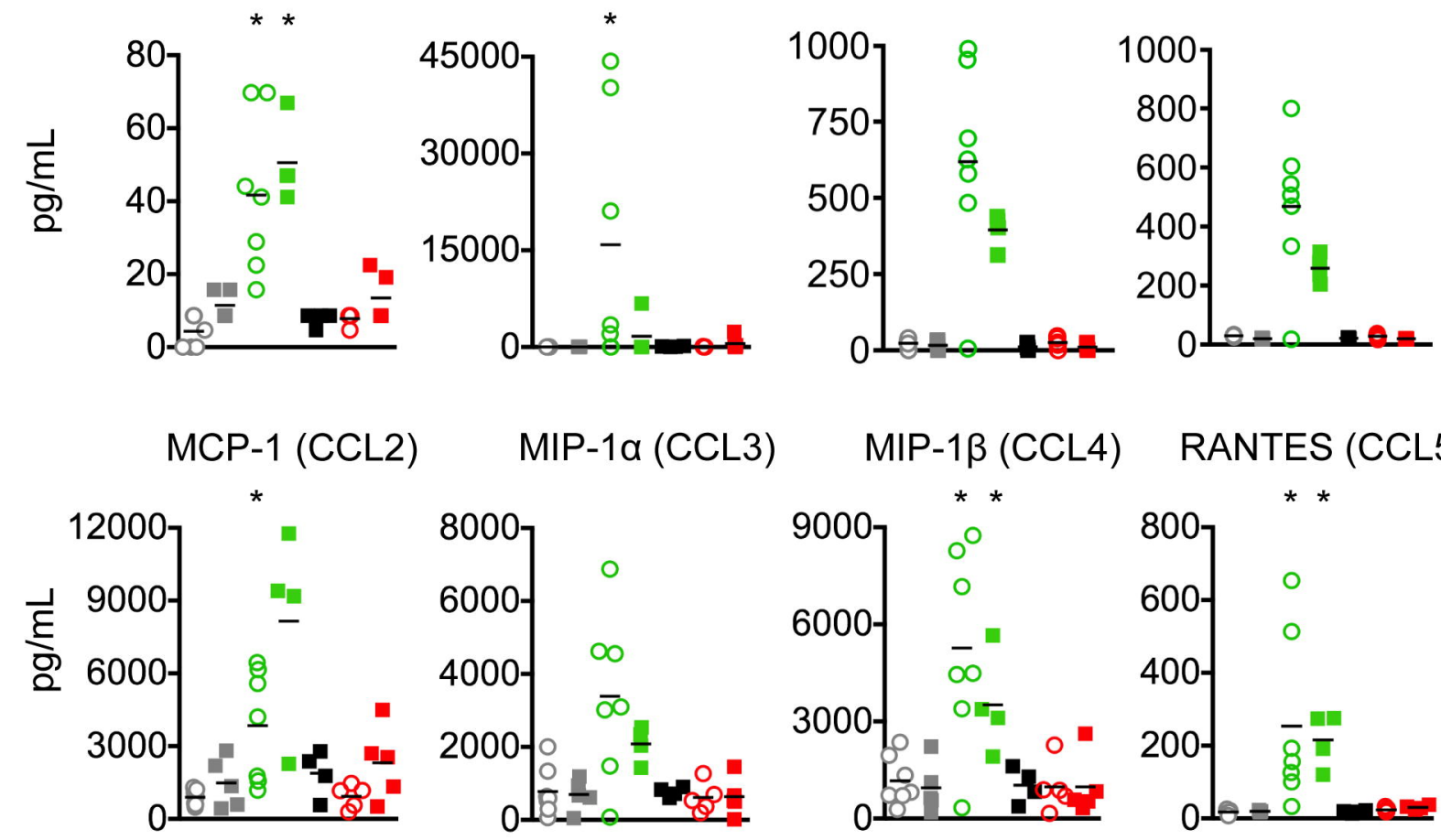

○ $12 \mathrm{hr}$

- $24 \mathrm{hr}$

- Mock

RIG-I agonist

- UV-WNV

WNV
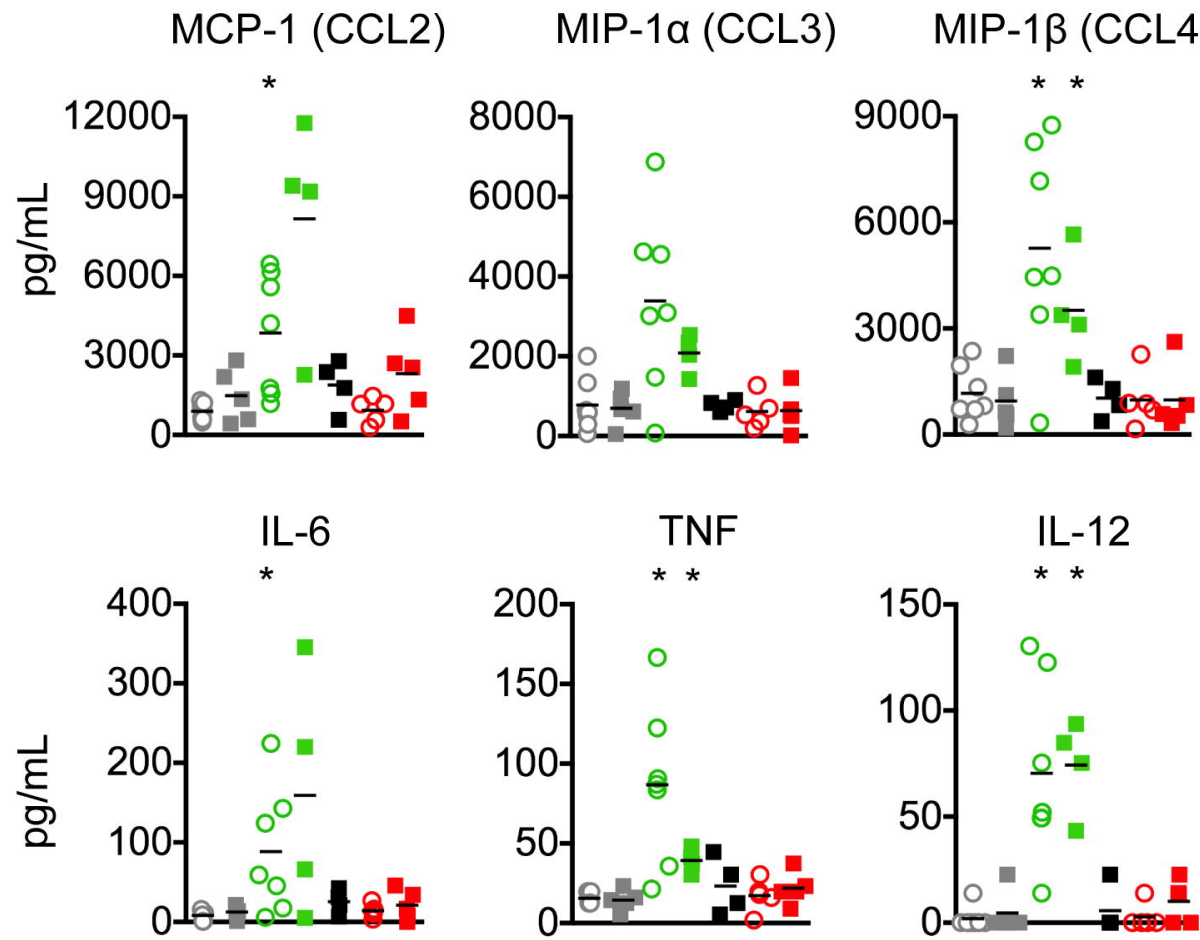

\section{RANTES (CCL5)}



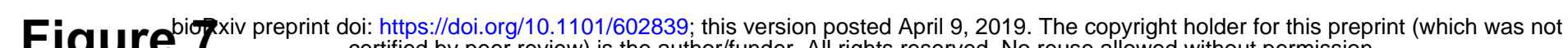
certified by peer review) is the author/funder. All rights reserved. No reuse allowed without permission.

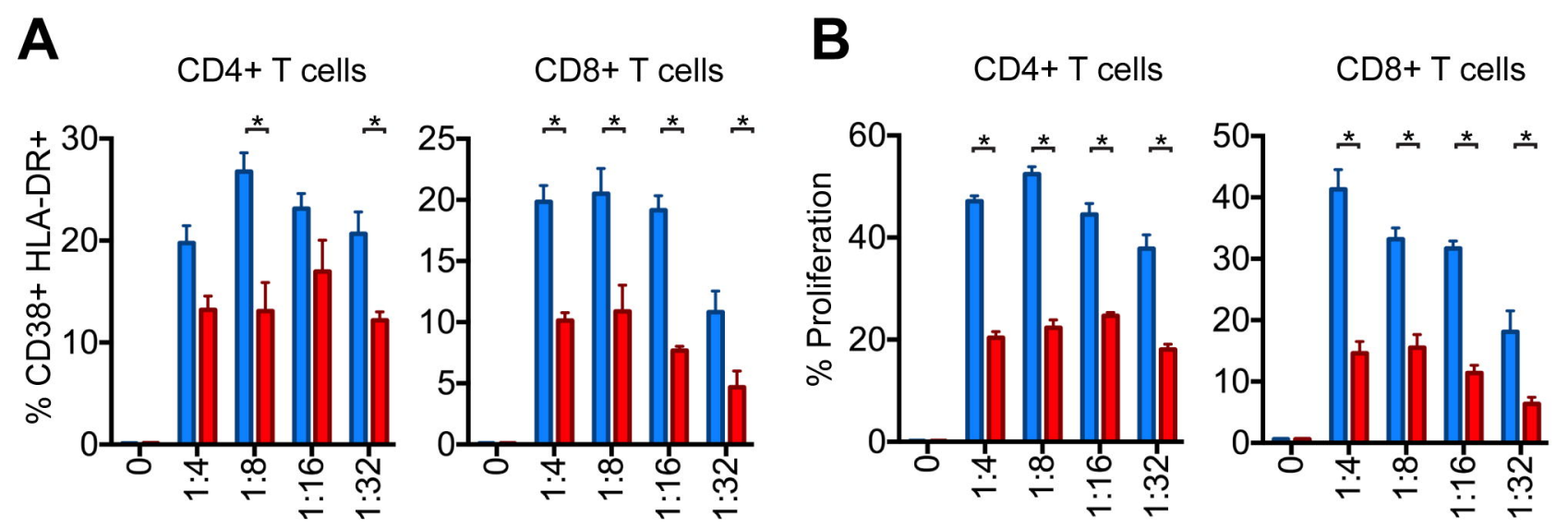

$D C: T$ cell ratio 